\title{
Dynamic of the organisation and structural inertia
}

\author{
CHRISTIAN PONCET*
}

\begin{abstract}
The story of organisation is often related as a successive period of stability and crisis. This article is based on the hypothesis that it is the intra-organisational inertia (i.e.: the organisation lack of adaptation to any modifications of the environment) the cause of usual severe ruptures in the evolution of firms. Indeed if we consider as theorists of organisations that the interrelation settled between the organisation and its environment is structural, the questions of representation, the evolution and adaptation become essential to understand the dynamic organisation. Starting by a geometric construction a model describing the organisation dynamics will be introduced, with elements enabling us to understand what an organisational change is (construction of a taxonomy) by the forces that generate it (construction of a dynamic). It will be more precisely question of trying to identify the notion of force compared with the notion of efficiency. And taking into account "this idea of crisis" which characterise all organisational transformation a geometric explanation based on the rupture of the organisation trajectory and singularity will be suggested. The interpretation of the first results thanks to a mere example of a model application in dynamic organisation brings to the fore, the importance of its history (for instance training in this example) in its adaptation capacity proving that it may become the cause of inertia, and organisational inefficiency.
\end{abstract}

Classification Codes: L21.

\section{Introduction}

Any organisation evolves and changes in an unceasingly changing environment. Although the representation of organisations has been the subject of theoretical, empirical and historical work, the dynamic approaches often remain at a descriptive stage ${ }^{1}$. The dynamic notion in organisations refers to changes in structures as well as to the rhythm of this change. These transformations can only be conceived as the result of interactions between internal organisation parameters and between the organisation and its environment. Interactions are therefore analysed as a set of complex variable games, previously identified, describing organisational structures as well as the context in which

* LASER/CREDEN, University of Montpellier I, Av. de la Mer, BP 9606, 34054 Montpellier Cedex, France. E-mail: poncet@sceco.univ-montp1.fr

Keywords: Organisation, structure, dynamic, space of representation.

${ }^{1}$ Chandler (1988), Labourdette (1992), Miller (1993), Morgan (1989), Woodward (1965).

(c) EDP Sciences 2001 
they are evolving. The way these (essentially qualitative) variables inter-react imposes a rhythm in changes, and this rhythm contains a transformation rate and depth? One can wonder what exactly changes and which coherence explain the need of a reorganisation in a firm. The organisation theories often tend to specify the contents of flow that animate them and structure them. In this perspective we can say that this article finds its bases in these fundamental contributions of the authors who mark the school of thought in organisation theories. This paper will consist in extending this work to suggest a framework of study of the organisation dynamic. However a dynamic approach of the organisation implies a preliminary highlighting of reference mark making it possible to appreciate the movement (i.e. change of the structures), but also the identification of a force that generates it. Lastly a univocal relation between the force and the movement must exist, the movement must be the exclusive effect of the application of the force. The geometrical representation of the organisational form is registered like the first stage of a construction of which the representation of a dynamics constitutes the objective. If we represent the organisation by a point located in an adequate space we can wonder how it is possible to explain organisational dynamic which is made up of prosperity moment as well as crisis and ruptures in its development? Any displacement in this space implies a transformation in the organisational form. In the second stage, I will suggest a dynamic model, that is to say identifying the force which applies to organisation and generates a modification in its structures and therefore, a displacement of the point built beforehand. How is then the trajectory of this point in this space shape (continuity, singularity)?

\section{A geometrical representation of the organisational structure}

A fundamental hypothesis on which is based the continuation from this representation lies in the possibility of representing on a given scale a priori the qualitative variables that will be introduced. Thus we will formulate this first assumption:

H1: The variables used to define the organisation and its environmental conditions are calibrated starting from a scale bounded by 0 and 1 .

This first assumption thus supposes that, on the one hand, any investigation carried out in an organisation makes it possible to identify these qualitative variables, and on the other hand, that the latter can rigorously result in a figure reflecting the degree of intensity with which this variable applies. We can therefore notice that such thought process often introduces irreducible dodges even if it has largely been used by organisation theorists.

\subsection{Theories of organisations as starting point}

Although being inspired, in its broad outline, by the construction of organisation proposed by Mintzberg ${ }^{2}$, the presentation of its model will not be taken into account here.

\footnotetext{
${ }^{2}$ Mintzberg (1982).
} 
We will simply recall some proposals he suggests and which will be useful in the elaboration of the model. This representation is also inspired by the ideas developed by the author and that will result in a geometrical model representing the organisational structures. This first part thus develops around an approach of a taxonomic-type, which will thereafter enable the construction of the dynamic of organisations. Like Mintzberg, we will identify the internal structures of the organisation starting from the design parameter. The element of the environment, for the organisation, which acts on certain structural aspects, will be presented in the form of factors of contingency. The integration of these two dimensions in the model, takes place in the following way:

\section{* Design parameters}

Considering that $\mathcal{P}$ is a vectorial space of dimension $n$ that will be called "space of the parameters". An organisational form will be represented by a linear combination of $n$ parameters. In fact on a scale of size built a priori, comes the second hypothesis:

H2: In the space $\mathcal{P}$, the design parameters constitute a basis and a point in this space identifies each organisational form studied ${ }^{3}$

The construction of such space supposes, of course, that the design parameters meet certain conditions, in particular that the vectors representing them check well the criteria of independence and genericity. $\mathbf{p}_{i}$ is the vector representing parameter $i$ in space $\mathcal{P}$, then any organisational configuration is written as $C^{j}$ bellow:

$$
C^{j}=\sum_{i=1}^{n} c_{i}^{j} \mathbf{p}_{i}
$$

design parameter thus arise as the canonical base ${ }^{4}$ of $n$-dimensional space $\mathcal{P}$. If it is initially added that $c_{i}^{j}$ can take either the values 0 , or value 1 , then each configuration admits or not a parameter given in its representation compared to the base of $\mathcal{P}$. From these definitions the configurations obtained express a form of ideality, since they represent the extreme demonstration of the intervention of design parameters in the definition of a configuration. As for any representation, and a fortiori geometrical, the organisational constructions suggested are based on a idealisation of the form which is inspired here by the "idealtype" as defined by Weber ${ }^{5}$ :

"The idealtype is just a representation, it is neither the historical reality nor the genuine reality, it serves even less as a diagram in which one could order reality as an example. Its signification only consists in giving a purely ideal limited concept which enables measuring reality to clarify the empirical contents of these elements and with which it is compared".

\footnotetext{
${ }^{3}$ According to us this hypothesis is implicitly contained in the Mintzberg organisational building (1982) (op. cit.).

${ }^{4}$ This means that we consider that the column vectors $\mathbf{p}_{i}$ of this base admit as components zeros except for line $i$, represented by value one.

${ }^{5}$ Weber (1992, p. 176).
} 
Each pure configuration obtained is represented as the result of a particular combination and without nuance of the design parameters. However, there are not as many configurations (of possible pure form) as potentially realisable combinations carried out by these parameters. In other words, the incompatibility of certain parameters between them will restrict the number of pure configurations. This geometrical construction is explicitly based on the same result reached by Mintzberg when he exclusively referred to the organisation theories. The model presented complies with this concept of "idealtype" and the joint contributions of the theorists and experts of organisations refer implicitly to this notion to identify and indicate an organisation.

\section{* Contingency factors}

After having constructed organisation by the identification of its internal structures, theorists were interested in the influence on their configuration, of the factors composing the environment. The contingency approach constitutes a significant stage in the evolution of the thought on the organisations, and it is essentially inspired by the work of Lawrence and Lorsch who conclude in these terms their studies ${ }^{6}$ :

"The internal data of the organisation, in term of structure and objectives, can be tested on their level of coherence with the variables of environment and the objectives of the individuals. The performance of the unity emerges as the result of this coherence".

As we demonstrated previously, the question is now to highlight a relation between the contingency factors, (defined as the whole of the external elements that act on the organisation) and the design parameters. This relation will be based on a third hypothesis:

H3: The "environment" generates an image of the organisation that we will call its "virtual form" corresponding to a perfectly effective design of the organisation. This virtual form is defined by the same design parameters as the form observed ${ }^{7}$.

Thus, the factors of contingency, identified as the relevant variables ${ }^{8}$ of the environment generate the design parameter of the virtual form ${ }^{9}$ of the organisation. By means of the "Action" there is thus a relation making it possible to pass from the factor of contingency to the virtual design parameters, i.e. those which, in this context would

\footnotetext{
${ }^{6}$ Lawrence and Lorsch (1989, op. cit., p. 192).

${ }^{7}$ It is here question of considering the theories of contingency at its extreme version and proving that the environment creates the organisation.

${ }^{8}$ In reference to the Crozier and Friedberg terminology (1977). "L'acteur et le système", which justifies this notion of pertinent environment (p. 140):

"[Pertinent environment] includes main social actors whose behaviours tend to condition directly the capacity of functioning of this organisation in a satisfactory way and achieving its goals without hiding us, mutatis-mutandis, that these objectives are not objective data but the product of a power struggle and bargaining which structure the system of the organisation subjacent action and its relations with its actors in its pertinent environment."

${ }^{9}$ In spite of the ambiguities of this term, the metaphor "virtual organisation" is often used in this paper in order to preserve the abstract point of view we confer to a perfectly effective image, projected starting from the environment on the structure of the organisation.
} 
generate the form of the perfectly effective organisation. In a first approximation, the environmental variables will be presented as exogenous parameters and will act on the design parameters, conceived in our approach like endogenous variables. Moreover the design parameters generate a "concrete" organisation, a form, which is revealed to the analyst who adopts this grid of reading. By what precedes it appears that the environment returns another image of the organisation, whose structures would answer in the most effective way the environmental contingencies. This "virtual organisation" is part of an extreme conception of the contingency theory; the case in which only the environmental constraints explain the structure of the organisation, and where the latter adapts to it instantaneously, in the absence of any consideration on the intra-organisational relations. The perfectly effective virtual form reacts in real time to all modifications of the environment whatever its sensitiveness. It appears, by what precedes, one second form for the organisation which, from a dynamic point of view, does not move under the same conditions as the preceding one (the observed structure). It is particularly not constrained by the rigidity of the observed existing organisational structures and therefore expresses no inertia in its evolution. Thus the trajectory followed by this "virtual form" is made conspicuous by its continuity in the space of the design parameters. The continuous character of the movement contrasts then with the generally discontinuous one followed by the organisational form built resulting from the parameters observed. This distinction generated in the evolution of the forms (between the virtual form and the observed form) will gradually induce a variation, a "distance", which will constitute an indicator of organisational effectiveness. Admitting that the search for the effectiveness represents the ultimate goal, the distance between the two organisational forms will generate a force that will be presented as the "engine" of the dynamic model. Its intensity will be proportional to the distance between the two forms. This means that the more the distance of the perfectly effective form is felt, the greater the need to bring together (restructuration etc.)

\subsection{Dynamic of the organisational structures}

The need to set up a relation between the factors of contingency and this virtual form in the space $\mathcal{P}$ built previously remains. In order to simplify the presentation, the organisational structures will be expressed by three design parameters ${ }^{10}$ (extension to $n$ parameters without any analytical difficulty). In reference to the terminology of Mintzberg that means:

- $P_{1}$ Mechanisms of connection ${ }^{11}$;

\footnotetext{
10 This illustration is juste an example due to the fact that the coherence of the model of Mintzberg is based on the exhaustive aspect of the parameters of design, and taking into account these parameters as a whole can lead to the expression of structural configurations. However we do think that mental representation, even if they are wrong on a strict theory point of view can help to understand a model. Therefore, in a three dimension space we give this illustration a purely didactic objective.

${ }^{11}$ Mintzberg (1982, op. cit.; p. 155).

"The organisations have developed many mechanisms to encourage relations among people and these mechanisms can be incorporated in the formal structural."
} 
- $P_{2}$ Training ${ }^{12}$

- $P_{3}$ Centralisation (direct supervision) ${ }^{13}$.

Supposing that only these three parameters intervene to describe the structures of the organisations and that only two structural configurations emerge (for example, the adhocratie and the simple structure) which we will be respectively calling $C^{1}$ and $C^{2}$. In the light of Mintzberg ${ }^{14}$ works, the pure configurations are expressed in the space of the parameters starting from its canonical base $\left\{p_{1}, p_{2}, p_{3}\right\}$. The corresponding configurations are declined in the following way:

$$
\begin{array}{l|lll} 
& p_{1} & p_{2} & p_{3} \\
\hline C^{1} & 1 & 1 & 0 \\
C^{2} & 0 & 0 & 1
\end{array}
$$

And the expression of configurations in this base is written:

$$
C^{1}=\left[\begin{array}{l}
1 \\
1 \\
0
\end{array}\right] \text { and } C^{2}=\left[\begin{array}{l}
0 \\
0 \\
1
\end{array}\right]
$$

What, according to the preceding conventions, make it possible to represent these ideal configurations ("idealtype" in reference to Weber) in the space of parameters with three dimensions ( $c f$. Fig. 1).

From the representation provided by Figure 1, the only idealtypes, a priori possible are located on the nodes of the cube. However on the $2^{3}$ theoretically realisable configurations, only two seem able to emerge (as an example, the point D, on Fig. 1 appears inaccessible since it should integrate at the same time centralisation and mechanisms of connection; two relatively incompatible concepts to implement following the theory of Mintzberg). For each idealtype the parameters enter completely or not in all the definitions of the configuration. Which means that an ideal configuration needs to be represented only by one limited number of these parameters. To leave from this framework "idéatypical", limited by many aspects, it is necessary to soften some hypothesis which guided the preceding construction. However, the organisational configurations are presented like possibilities. In the space of the design parameters and in facts, certain combinations seem unrealisable (and this according to the internal principle of coherence of the organisation which excludes the appearance of certain "ideal" form. The configurations observed are consequently in a subspace $\boldsymbol{D}_{j}$ of $\boldsymbol{P}$ which includes the point representing the ideal form. In other words, for an organisational form $j$, the design

\footnotetext{
${ }^{12}$ Mintzberg (1982, op. cit.; p. 109).

"The word training will be used for the processes enabling education in professional life."

${ }^{13}$ Mintzberg (1982, op. cit.; p. 173).

"The structure is centralised when all the decision-making powers are situated in one point in the organisation, in extreme cases, in the hands of only one person. However we will say that the structure is decentralised when the power is shared among many people."

${ }^{14}$ Mintzberg (1982, op. cit.).
} 


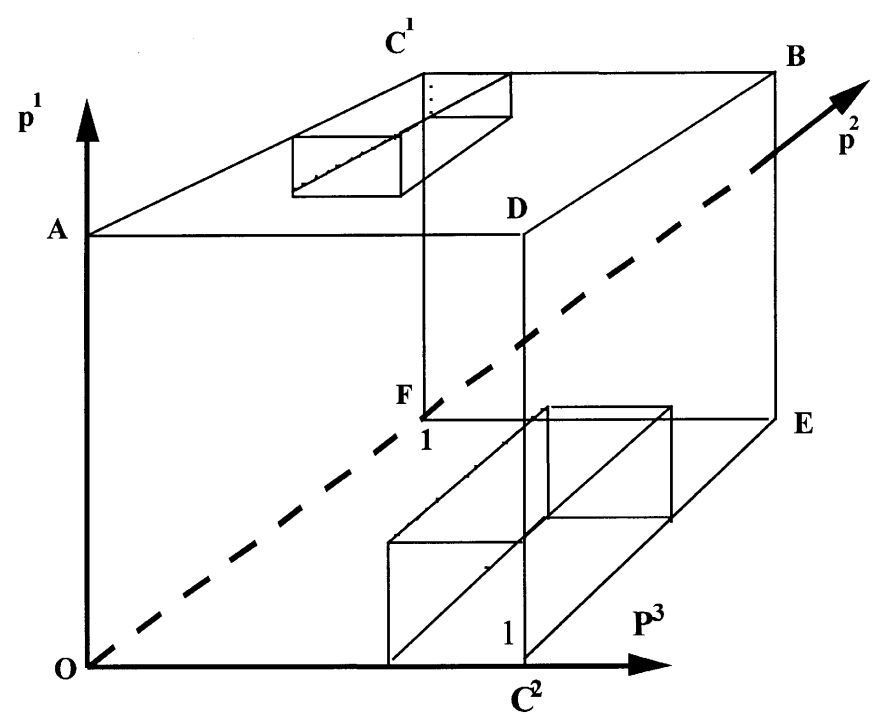

Fig. 1. A representation of the configurations in the space of parameters.

parameters $P_{i}$ are not limited to values 0 or 1 they vary in fact in the interval [0-1] they are co-ordinated, respecting certain principle of coherence, which melt the structure of the organisation. From the preceding example, if one indicates by $C^{1}$ the simple structure (according to the terminology of Mintzberg), this denomination will apply to all forms which admits the characteristics:

- Much centralisation;

- Little training;

- Few mechanisms of connection.

Concretely, the organisational form comes up in a right-angled parallelepiped ${ }^{15}$ (Fig. 1), and in this set all the points will represent a combination of feasible parameters of conception and therefore coherent for the organisation. The construction of the borders of these sets depends on the level of analysis one may have. On the one hand an objective definition of these limits can be conceived starting for example from the strict technical relations that the parameters establish between each others due to their properties ${ }^{16}$. On the other hand borders defining a more reduced unit which would reveal a more $s u b$ jective conception of the capacities of adaptation of the organisation ${ }^{17}$. Lastly, this

\footnotetext{
${ }^{15}$ This representation which is the simplest one, can be prolonged by a relation (not linear) between the two parameters of design that would define a border with much more complex contours. Each organisation generating its own limits thanks to its history and own inertia.

${ }^{16}$ This position could for example reflect the vision of an external expert carrying out an audit on the organisation.

${ }^{17}$ This point is more precisely the vision of the leaders, who knowing the organisation (on historical, sociological and human point of view), certainly introduce more limited fields of evolution.
} 
presentation defines, according to the same logic, a set of inaccessible points. It thus refers to the field of the space $\mathcal{P}$, in which no observable configuration will be able to appear indeed.

The observable form, limited in its capacities of adaptation by the internal coherence of the design parameters ${ }^{18}$, is thus opposed to the virtual form, which moves without apparent constraint in $\mathcal{P}$ space. The factors of contingency, while acting on the virtual form, will widen a gap with the effective structure of the organisation. It thus remains to establish a formal relation between the factors of contingency and the virtual structure of the organisation, which means suggesting an expression of the environmental conditions in term of design parameters, for the virtual form. This construction is carried out in two stages ${ }^{19}$ :

-1- Let $\mathbf{S}$ be a vectorial subspace of $\boldsymbol{Z}$ that constitutes the relevant space of the factors of contingency. By identifying a base of this subspace, expressed according to the canonical base of 7 , it appears a first linear application $h_{1}:\left(7 \stackrel{h_{1}}{\longrightarrow} \mathbf{S}\right)$ of which the matrix $[\mathbf{A}]=\left\{\alpha_{i j}\right\}$. In such as any point $y$ of $\boldsymbol{Z}$ one associates a point $y^{\prime}$ of $\boldsymbol{S}$. The subspace $\boldsymbol{S}$ becomes thus the relevant environment of the organisation.

-2- Let an application $h_{2}$ of $\boldsymbol{S}$ in $\mathcal{P}$ defined by a matrix $[\mathbf{H}]=\left\{\eta_{i j}\right\}$ which in any point of $y^{\prime}$ of $\boldsymbol{S}$ associates a point $x^{\prime}$ of $\boldsymbol{P}$. The subspace $\boldsymbol{S}$ being defined as the relevant environment of the organisation, the application $h_{2}$ must necessarily be bijective. Consequently it constitutes an isomorphism of the vectorial space $S$ on the vectorial space $\mathcal{P}{ }^{20}$. That means in particular that the basis of the subspace $\boldsymbol{S}$ will have as an image by the application $h_{2}$ a base of space $\mathcal{P}$.

\section{From the movement of the virtual form to the dynamics of the organisation}

The step adopted until now leads, for a same organisation, to the construction of two points in a space $\mathcal{P}$ of the parameters. The relative position of these two points, at a given time, constitutes a criterion of organisational efficiency. Therefore, starting from the relative positioning of the point $x^{\prime}$ (observed form) and $x^{\prime}$ (virtual form) a force develops which will be all the more intense since the point $x^{\prime}$ moves away from $x$. The increased distance between these two points accentuates the efficiency of the organisation and consequently, makes all the more significant propensity to modify the structure of the organisation (Fig. 2).

\footnotetext{
${ }^{18}$ To these objective reasons are added power struggles tending to increase inertia within the organisation. The relation between the power and organisational inertia are clearly developed by Crozier and Friedberg (op. cit.).

${ }^{19}$ The formal development of these two stages is explained in Appendix I.

${ }^{20}$ The coherence of the model suggested by Mintzberg implicitly implies this bijective character.
} 


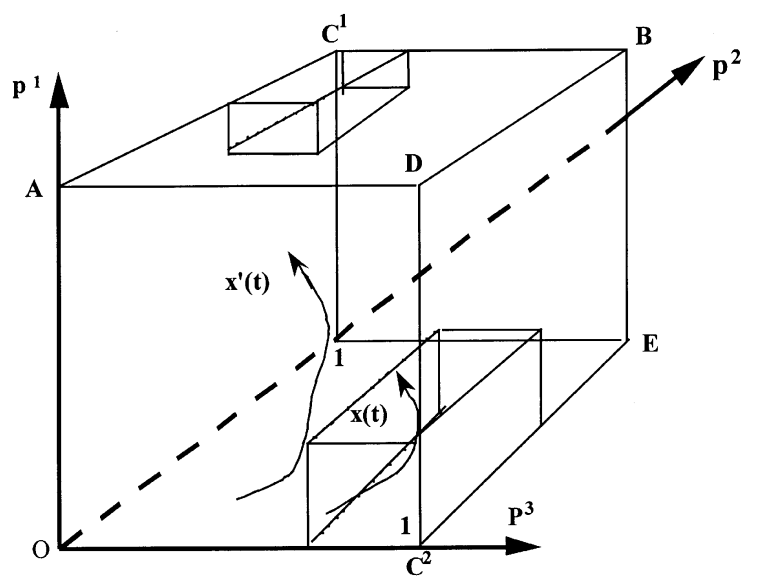

Fig. 2. Representation of the trajectories of items $x$ and $x^{\prime}$ in the space of parameters.

\subsection{The virtual form and its geometrical representation}

From what precedes, the model of the dynamics of the organisation will reach its target if one builds a relation between the factors of contingency and the virtual form of the organisation. This relation will be highlighted starting from a very simplified model, like previously:

- $p_{1}$ Mechanisms of connection;

- $p_{2}$ Training;

- $p_{3}$ Centralisation (direct supervision).

The factors of contingency selected arise for example in these terms:

- $f_{1}$ The sophistication of the technical system ${ }^{21}$;

- $f_{2}$ The hostility of the environment ${ }^{22}$;

- $f_{3}$ The complexity of the environment ${ }^{23}$;

- $f_{4}$ The size of the organisation ${ }^{24}$.

${ }^{21} f_{1}$ : The sophistication of the technical system considers as for as this subject is concerned the following hypothesis (as convergent results of a great numbers of studies carried out by various authors):

"hypothesis 7: the more the technical system is sophisticated, the more the administrative structure is elaborated, more precisely, more functional logistics are numerous and qualified more a selective decentralisation is operated at their advantage, the organisation using mechanisms of connection to co-ordinate their work."

${ }^{22} f_{2}$ : Hostility of the environment.

"hypothesis 12: an extreme hostility of its environment leads any organisation to centralise its structure in a temporary way."

${ }^{23} f_{3}$ : The complexity of the environment.

"hypothesis 10: more the environment is complex and more the structure is decentralised."

${ }^{24} f_{4}$ : The size of the organisation.

"The entrepreneurial stage brings a vertical division of work with a manager who takes himself all the main decisions, co-ordinates and supervise them directly, all other members of the organisation executing the required instructions." 
Consider a combination of the factors of contingency that expresses the maximum influence that these variables exert on a particular design parameter. There are thus values reached by factors $\left\{y_{j} ; j=1 \ldots 4\right\}$ which would lead a design parameter $x_{i}^{\prime}$ to be expressed fully in the normed base of $\boldsymbol{P}$. Hence let the triplet of vectors $\left\{s_{1}^{\prime}, s_{2}^{\prime}, s_{3}^{\prime}\right\}$, representing in a 4-dimension space $\boldsymbol{Z}$ the factors of contingency, leading to a maximal expression of parameters $\left\{p_{1}, p_{2}, p_{3}\right\}$. The triplet $\left\{s_{1}^{\prime}, s_{2}^{\prime}, s_{3}^{\prime}\right\}$ constituting in theory a base of subspace $S$ (provided one demonstrates the independence of these vectors in the space 7).

$$
\left\{s_{1}^{\prime}=\left[\begin{array}{c}
1 \\
\sigma_{1}^{2} \\
1 \\
\sigma_{1}^{4}
\end{array}\right] ; \quad s_{2}^{\prime}=\left[\begin{array}{c}
\sigma_{2}^{1} \\
0 \\
1 \\
\sigma_{2}^{4}
\end{array}\right] ; \quad s_{3}^{\prime}=\left[\begin{array}{l}
0 \\
1 \\
0 \\
1
\end{array}\right]\right\} .
$$

For example, the first term translates that, if the sophistication of the technical system and the complexity of the environment reach their maximum level and if the hostility of the environment and the size of the organisation ${ }^{25}$, take an adequate value, then the mechanism of connection in a perfectly effective organisation will have to reach their maximal level. Assuming this hypothesis, the triplet $\left\{s_{1}^{\prime}, s_{2}^{\prime}, s_{3}^{\prime}\right\}$ by the application $h_{1}{ }^{26}$ will thus have as an image in $\mathbf{S}$ :

$$
\left\{s_{1}=\left[\begin{array}{c}
1 \\
1 \\
\sigma_{1}^{4}
\end{array}\right] ; s_{2}=\left[\begin{array}{c}
\sigma_{2}^{1} \\
1 \\
\sigma_{2}^{4}
\end{array}\right] ; s_{3}=\left[\begin{array}{l}
0 \\
0 \\
1
\end{array}\right]\right\} .
$$

It results that appears a new base of the subspace $\boldsymbol{S}$, in the condition of course that these vectors are linearly independent. What amounts to writing that:

$$
\Delta_{\Sigma}=1-\sigma_{2}^{1} \neq 0
$$

The relation above is well checked since, by definition, we necessarily have $0<\sigma_{2}^{1}<1$. Which means that the level reached by the "sophistication of the technical system" and which enables observing a maximum degree of "training" in the organisation must be lower than that of the "complexity of the environment". This, in other words, bring to the conclusion that it is the "complexity of the environment" which influences more the

\footnotetext{
${ }^{25}$ The calibration of this value we shall retain that $\sigma_{1}^{4}$ approaches 1 for a minimum size of the organisation and tends towards 0 when the latter becomes very large. This index is thus decreasing with the size of the organisation.

${ }^{26}$ Please refer to Appendix I for a matrix specification of this application.
} 
level of "training" in the organisation. The matrix determinant above is strictly positive, consequently in the subspace $\boldsymbol{S}$, any vector will be expressed according to the base $\left\{s_{1}, s_{2}, s_{3}\right\} \in S$. Under these conditions, the relevant factors of the environment of the organisation make it possible to define this subspace $S$ that admits as basis a combination of the factors leading to the full expression of each design parameter. Any environmental condition will arise consequently in the form:

$$
y=y_{1}^{\prime} s_{1}+y_{2}^{\prime} s_{2}+y_{3}^{\prime} s_{3} .
$$

Moreover, from an intra-organisational point of view, so that the expression of a design parameter reaches in the organisation its maximal level (equal 1), it is necessary that the other parameters observe certain conditions. More generally, consider $\pi_{k}^{i}$ the level that the design parameter $i$ must reach so that the parameter $k$ can express fully (that is to say equal 1) it follows that:

$$
\pi_{k}^{i}=1
$$

The components of the triplet: $\left\{\pi_{1}, \pi_{2}, \pi_{3}\right\}$ define a new base in $\mathcal{P}$ with for example:

$$
\pi_{1}=\left[\begin{array}{c}
1 \\
\pi_{1}^{2} \\
0
\end{array}\right] ; \pi_{2}=\left[\begin{array}{c}
\pi_{2}^{1} \\
1 \\
\pi_{2}^{3}
\end{array}\right] ; \pi_{3}=\left[\begin{array}{c}
0 \\
\pi_{3}^{2} \\
1
\end{array}\right] .
$$

What for $\pi_{1}$ is interpreted in the following way: so that the mechanisms of connection in the organisation reach their maximal level (equal 1) it is necessary that the degree of training rises $\pi_{1}^{2}$ and that centralisation be null. This triplet $\left\{\pi_{1}, \pi_{2}, \pi_{3}\right\}$ constitutes a base in $P$ :

$$
1-\pi_{1}^{2} \pi_{2}^{1}-\pi_{2}^{3} \pi_{3}^{2} \neq 0 \Leftrightarrow \pi_{1}^{2} \pi_{2}^{1}+\pi_{2}^{3} \pi_{3}^{2} \neq 1
$$

However, taking into account the definition of the parameters, which compose these matrices:

$$
1-\pi_{1}^{2} \pi_{2}^{1}-\pi_{2}^{3} \pi_{3}^{2}>0
$$

Indeed by interpreting the product: $\pi_{i}^{j} \pi_{j}^{i}$, as the level which must at least reach the parameter $j$ in order that of parameter $i$ rises with $\pi_{j}^{i}$. Consequently $\pi_{1}^{2} \pi_{2}^{1}$ and $\pi_{3}^{2} \pi_{2}^{3}$ will represent the levels of training implemented so that the mechanisms of connection and centralisation respectively rise with $\pi_{2}^{1}$ and $\pi_{2}^{3}$, i.e., the threshold which they must reach to allow the maximum expression of the "training" (thus equalises 1) ${ }^{27}$.

\footnotetext{
27 That means that to reach a maximum level of "training" it is necessary that the mechanisms of connection and "centralisation" reach a certain value. However to reach this level it is also necessary to implement a certain level of "training".
} 
Consequently, the determinant of the matrix will be interpreted like the level of "training", entirely directed towards the processing of the environment and not employed to implement in-house the "mechanisms of connection" and "centralisation" necessary to the maximum expression of this "training". By bringing this process closer to the concept of apprenticeship, that returns for the organisation to release a sufficient potential of training, therefore answering in a perfectly effective way the constraints imposed by the environment. The organisation must previously adapt in-house the adequate design parameters to be able to express this training level. For these reasons, this determinant must necessarily be strictly positive.

Consider thus the matrices:

$$
[\mathbf{S}]=\left[\begin{array}{ccc}
1 & \sigma_{2}^{1} & 0 \\
1 & 1 & 0 \\
\sigma_{1}^{4} & \sigma_{2}^{4} & 1
\end{array}\right] \text { and }[\Pi]=\left[\begin{array}{ccc}
1 & \pi_{2}^{1} & 0 \\
\pi_{1}^{2} & 1 & \pi_{3}^{2} \\
0 & \pi_{2}^{3} & 1
\end{array}\right]
$$

The coefficient of the matrix [S] will play a relay role between the factors of contingency and the design parameters. Those of the matrix [П] will take part in the internal coherence of the organisation in this "perfectly effective" context. Considering the matrix $[\Sigma]$ as the matrix transposed of $[\mathbf{S}]$, the application $h_{2}$ will be represented by the matrix $[\mathbf{H}]$ such as ${ }^{28}$ :

This result enables expressing the coefficients of the matrix $[\mathbf{H}]$ :

$$
[\mathbf{H}]=[\Pi]^{-1}[\Sigma]
$$

with:

$$
[\Pi]^{-1}=\frac{1}{\Delta_{\Pi}}\left[\begin{array}{ccc}
1-\pi_{2}^{3} \pi_{3}^{2} & -\pi_{2}^{1} & \pi_{2}^{1} \pi_{3}^{2} \\
-\pi_{1}^{2} & 1 & -\pi_{3}^{2} \\
\pi_{1}^{2} \pi_{2}^{3} & -\pi_{2}^{3} & 1-\pi_{1}^{2} \pi_{2}^{1}
\end{array}\right]
$$

and

$$
\Delta_{\Pi}=1-\pi_{1}^{2} \pi_{2}^{1}-\pi_{2}^{3} \pi_{3}^{2}>0
$$

Consequently

$$
[\mathbf{H}]=\frac{1}{\Delta_{\Pi}}\left[\begin{array}{ccc}
1-\pi_{2}^{3} \pi_{3}^{2}-\sigma_{2}^{1} \pi_{2}^{1} & 1-\pi_{2}^{3} \pi_{3}^{2}-\pi_{2}^{1} & \sigma_{1}^{4}\left(1-\pi_{2}^{3} \pi_{3}^{2}\right)-\sigma_{2}^{4} \pi_{2}^{1}+\pi_{2}^{1} \pi_{3}^{2} \\
\sigma_{2}^{1}-\pi_{1}^{2} & 1-\pi_{1}^{2} & -\sigma_{1}^{4} \pi_{1}^{2}+\sigma_{2}^{4} \pi_{3}^{2} \\
-\pi_{2}^{3}\left(\sigma_{2}^{1}-\pi_{1}^{2}\right) & -\pi_{2}^{3}\left(1-\pi_{1}^{2}\right) & \sigma_{1}^{4} \pi_{1}^{2} \pi_{2}^{3}-\sigma_{2}^{4} \pi_{2}^{3}+\left(1-\pi_{1}^{2} \pi_{2}^{1}\right)
\end{array}\right]
$$

${ }^{28}$ Please refer to Appendix II for details concerning the demonstration. 
to conclude

$$
\begin{aligned}
& x_{1}^{\prime}=\left[\frac{1-\pi_{2}^{3} \pi_{3}^{2}-\sigma_{2}^{1}}{1-\pi_{2}^{3} \pi_{3}^{2}-\pi_{1}^{2} \pi_{2}^{1}}\right] y_{1}^{\prime}+\left[\frac{1-\pi_{2}^{3} \pi_{3}^{2}-\pi_{2}^{1}}{1-\pi_{2}^{3} \pi_{3}^{2}-\pi_{1}^{2} \pi_{2}^{1}}\right] y_{2}^{\prime}-\left[\frac{\pi_{2}^{1}\left(\sigma_{2}^{4}-\pi_{3}^{2}\right)-\sigma_{1}^{4}\left(1-\pi_{2}^{3} \pi_{3}^{2}\right)}{1-\pi_{2}^{3} \pi_{3}^{2}-\pi_{1}^{2} \pi_{2}^{1}}\right] y_{3}^{\prime} \\
& x_{2}^{\prime}=\left[\frac{\sigma_{2}^{1}-\pi_{1}^{2}}{1-\pi_{2}^{3} \pi_{3}^{2}-\pi_{1}^{2} \pi_{2}^{1}}\right] y_{1}^{\prime}+\left[\frac{1-\pi_{1}^{2}}{1-\pi_{2}^{3} \pi_{3}^{2}-\pi_{1}^{2} \pi_{2}^{1}}\right] y_{2}^{\prime}-\left[\frac{\sigma_{1}^{4}-\pi_{1}^{2}-\sigma_{2}^{4}+\pi_{3}^{2}}{1-\pi_{2}^{3} \pi_{3}^{2}-\pi_{1}^{2} \pi_{2}^{1}}\right] y_{3}^{\prime} \\
& x_{3}^{\prime}=\left[\frac{-\pi_{2}^{3}\left(\sigma_{2}^{1}-\pi_{1}^{2}\right)}{1-\pi_{2}^{3} \pi_{3}^{2}-\pi_{1}^{2} \pi_{2}^{1}}\right] y_{1}^{\prime}+\left[\frac{\pi_{2}^{3}\left(1-\pi_{1}^{2}\right)}{1-\pi_{2}^{3} \pi_{3}^{2}-\pi_{1}^{2} \pi_{2}^{1}}\right] y_{2}^{\prime}+\left[\frac{\pi_{2}^{3}\left(\sigma_{1}^{4} \pi_{1}^{2}-\sigma_{2}^{4}\right)+\left(1-\pi_{1}^{2} \pi_{2}^{1}\right)}{1-\pi_{2}^{3} \pi_{3}^{2}-\pi_{1}^{2} \pi_{2}^{1}}\right] y_{3}^{\prime}
\end{aligned}
$$

This expression shows an explicit linear relation between the factors of contingency and the design parameters (applied to a virtual organisation, therefore perfectly effective).

\subsection{Interpretation of the results and the analytical prospects}

In relation with the initial targets, the comments which rise from this relation must answer two questions: on the one hand, showing the coherence of the results obtained with those of the theories of the organisations, on the other hand, interpreting these results as a stage in the construction of dynamic models for the organisations. (This prospect will be considered in the conclusion.)

As far as the coherence of the model is concerned, this results show that the signs which affect each variable $y_{i}^{\prime}$ corroborate a priori many intuitive relations. Thus, the "mechanisms of connection" give a positive answer to the two factors of contingency: "sophistication of the technical system", "complexity of the environment" and negatively to "the size of the organisation" (by admitting that: $\left.\sigma_{1}^{4} \approx 0\right){ }^{29}$. On the other hand, "the centralisation of the decisions" is a decreasing function of the complexity of the environment as well as "the size of the organisation". Concerning the influence of "the sophistication of the technical system" everything will depend on the comparison in value on $\pi_{1}^{2}$ with $\sigma_{2}^{1}{ }^{30}$. Moreover, the decomposition, for example, of the second equation enables refining theses interpretations (the principle of decomposition developed here applies to the other equations by adapting first the comments that will follow). This second equation can be thus written in the following form:

$$
\left(1-\pi_{2}^{3} \pi_{3}^{2}-\pi_{1}^{2} \pi_{2}^{1}\right) x_{2}^{\prime}=\left(\sigma_{2}^{1} y_{1}^{\prime}+y_{2}^{\prime}+\sigma_{2}^{4} y_{3}^{\prime}\right)-\pi_{1}^{2}\left(y_{1}^{\prime}+y_{2}^{\prime}+\sigma_{1}^{4} y_{3}^{\prime}\right)-\pi_{3}^{2} y_{3}^{\prime}
$$

\footnotetext{
29 "The mechanisms of design" will be fully expressed if the "size of the organisation" proves to be the largest one.

${ }^{30}$ Given that the denominator of these expressions is established according to the "training" base, reconstructing it when dealing with the first equation (mechanism of connection) or the third one (centralisation) is advisable. These extrapolations generating no difficulties.
} 
consequently, with a matrix is written:

$$
\left(1-\pi_{2}^{3} \pi_{3}^{2}-\pi_{1}^{2} \pi_{2}^{1}\right) x_{2}^{\prime}=\left[\begin{array}{lll}
\sigma_{2}^{1} & 1 & \sigma_{2}^{4}
\end{array}\right]\left[\begin{array}{l}
y_{1}^{\prime} \\
y_{2}^{\prime} \\
y_{3}^{\prime}
\end{array}\right]-\pi_{1}^{2}\left[\begin{array}{lll}
1 & 1 & \sigma_{1}^{4}
\end{array}\right]\left[\begin{array}{l}
y_{1}^{\prime} \\
y_{2}^{\prime} \\
y_{3}^{\prime}
\end{array}\right]-\pi_{3}^{2}\left[\begin{array}{lll}
0 & 0 & 1
\end{array}\right]\left[\begin{array}{l}
y_{1}^{\prime} \\
y_{2}^{\prime} \\
y_{3}^{\prime}
\end{array}\right]
$$

or with the matrix notations used before:

$$
x_{2}^{\prime}-\left(\pi_{2}^{3} \pi_{3}^{2}+\pi_{1}^{2} \pi_{2}^{1}\right) x_{2}^{\prime}=\left[\sigma_{2}\right]\left[y^{\prime}\right]-\pi_{1}^{2}\left[\sigma_{1}\right]\left[y^{\prime}\right]-\pi_{3}^{2}\left[\sigma_{3}\right]\left[y^{\prime}\right] .
$$

The two members of the expression above refer to the same organisational object:

- the virtual design parameters (and thus of internal coherence in the organisation) on the left side member,

- the action of the factors of contingency and thus of the adequacy of the structure of this "virtual" organisation to its environment), in the right-hand side member.

- The left member: $x_{2}^{\prime}-\left(\pi_{2}^{3} \pi_{3}^{2}+\pi_{1}^{2} \pi_{2}^{1}\right) x_{2}^{\prime}$ represents the level $x_{2}^{\prime}$ reached by the "training" and, so that this training level be reached a certain degree of mechanism of connection and of centralisation is necessary. Thus in the "perfectly effective" organisation, if the environmental conditions require that the level of training must rise to $x_{2}^{\prime}$ it will be reached only if there exists some "mechanism of connection" sufficient to manage it. (this level must reach to $\pi_{2}^{1}\left(\pi_{2}^{1} x_{2}^{\prime}\right)$ ). Moreover, all "training" require a certain level of "centralisation" and to satisfy this constraint, it is also necessary to release "training" for this purpose (thus, to implement a training level $x_{2}^{\prime}$, it is necessary to mobilise $\pi_{3}^{2}\left(\pi_{2}^{3} x_{2}^{\prime}\right)$ to manage centralisation). If within this framework, the environment (by means of contingency factor actions) leads the organisation to release a level $x_{2}^{\prime}$, a part $\left(\pi_{2}^{3} \pi_{3}^{2}+\pi_{1}^{2} \pi_{2}^{1}\right) x_{2}^{\prime}$ will be used for management of the other parameters to be able to achieve this goal. Therefore only the expression $x_{2}^{\prime}-\left(\pi_{2}^{3} \pi_{3}^{2}+\pi_{1}^{2} \pi_{2}^{1}\right) x_{2}^{\prime}$ will remain assigned to the specific processing of the environment.

- The member of the right-hand side (which results in the matrix expression: $\left[\sigma_{2}\right]\left[y^{\prime}\right]-$ $\pi_{1}^{2}\left[\sigma_{1}\right]\left[y^{\prime}\right]-\pi_{3}^{2}\left[\sigma_{3}\right]\left[y^{\prime}\right]$ admits exactly the same principles of interpretation, but starting from the factors of contingency. Since the vector $\left[\sigma_{2}\right]$ is composed of the coefficients (factors of contingency) which lead the "training" to be fully expressed in the organisation, the matrix product $\left[\sigma_{2}\right]\left[y^{\prime}\right]$ will translate the level of training that should reach the organisation in the context defined, starting from the factors of contingency $\left[y^{\prime}\right]$. In the same order of idea, and under the given environmental conditions given by $\left[y^{\prime}\right]$ to give a "perfectly effective" organisational response in this context, the "mechanisms of connection" must reach the level $\left[\sigma_{1}\right]\left[y^{\prime}\right]$. However, to reach this degree, the organisation will have to mobilise "training" on a level $\pi_{1}^{2}\left[\sigma_{1}\right]\left[y^{\prime}\right]$. An identical conception applied to "centralisation" makes it possible to conclude that, to reach the required threshold the organisation must call $\pi_{3}^{2}\left[\sigma_{3}\right]\left[y^{\prime}\right]$ upon some "training". Thus to answer to these environment constraints (analyse starting from factor of contingency). The organisation will mobilise a level $\left[\sigma_{2}\right]\left[y^{\prime}\right]$ of "training". This mobilisation is partly affected to support the "mechanism of connection" and the "centralisation" to enable 
these parameters to answer in a "perfectly effective" manner to the external conditions $\left(\pi_{1}^{2}\left[\sigma_{1}\right]\left[y^{\prime}\right]+\pi_{3}^{2}\left[\sigma_{3}\right]\left[y^{\prime}\right]\right)$. The rest being directly affected to the environment processing $\left(\left[\sigma_{2}\right]\left[y^{\prime}\right]-\pi_{1}^{2}\left[\sigma_{1}\right]\left[y^{\prime}\right]-\pi_{3}^{2}\left[\sigma_{3}\right]\left[y^{\prime}\right]\right)$.

In conclusion, equality between two members is possible due to the "perfectly effective" organisation, this result in an adequate and instantaneous adaptation of the structures to the environmental conditions. This equality represents another conception of the "perfectly effective" structure. In other words, this virtual form will reach the perfectly effective degree of training in a given environment if the organisation is previously capable to mobilise instantaneously a sufficient level of other parameters. This condition reveals the importance of a previous training process allowing the entire expression of this training level. Opposing the observed form to the perfectly effective form emerges a new cause in the organisational inertia that is to say the necessary time for training in the observed form, which will increase the distance with the virtual form, for which by definition, this training period does not exist.

\section{Conclusion}

The presentation of this model intends to consider the dynamics of the organisation as a search for effectiveness in the environment. This effectiveness is inversely proportional to the distance which, in the reference mark constituted by the design parameters, separates the observed form from the virtual form. The virtual form of the organisation is expressed in a univocal way, and thus this organisation conceived is assimilated to an image that projects the environment. It immediately finds its expression in terms of design parameters in the space $\boldsymbol{P}$. Time has not the same impact on the two forms because the trajectory of the virtual form a priori continuously evolves in all the $\mathcal{P}$ space, whereas the observed form is enclosed and limited (in limits defined by the compatibility of the design parameters between each others). It moreover contains inertia, which limits its rate of adaptation. These two constraints lead to a radical opposition in the way the two forms are expressed. In fact, considering that each organisational configuration is defined in $\boldsymbol{D}_{i} ; \boldsymbol{D}_{i} \subset \mathcal{P}$ (represented on Fig. 1 by a right-angled parallelepiped):

-1- If the proximity between the virtual form and the field $\boldsymbol{D}_{i}$, in which the observed structure is, seems strong enough, the organisation will adapt to its environment bringing limited modifications of certain design parameters. The trajectory in the space $D_{i}$ of the observed form will be continuously differentiable and thus the organisation will not reach a crisis by maintaining an "acceptable" level of effectiveness.

-2- If the virtual form slightly moves away from the $\boldsymbol{D}_{i}$ field, in which the organisation is, its minor adaptation will confront it to the borders of this field. It will consequently not longer be able to follow continuously this virtual form. With the increasing distance, internal conflicts tend to rise, leading to a deep crisis in the organisation. This crisis will graphically be illustrated by a "jump" from the $\boldsymbol{D}_{i}$ field to the field $\boldsymbol{D}_{k}$ (therefore a structural configuration). This phenomenon can therefore 
have a formal representation by the catastrophe theories ${ }^{31}$, in which effectiveness conceived as a distance would constitute a variable of "control". As if a "spring" existed between the virtual form and the observed structure, tensions due to the continuous distance of the former leading it to brutally come out from its field to place itself in a closer field. This "catastrophic jump" of the organisation, this singularity in the representation of its trajectory finds an internal expression by the idea of crisis, followed by an organisational restructuration.

\section{Appendix I: Construction of the matrix of passage of the application $h_{1}$}

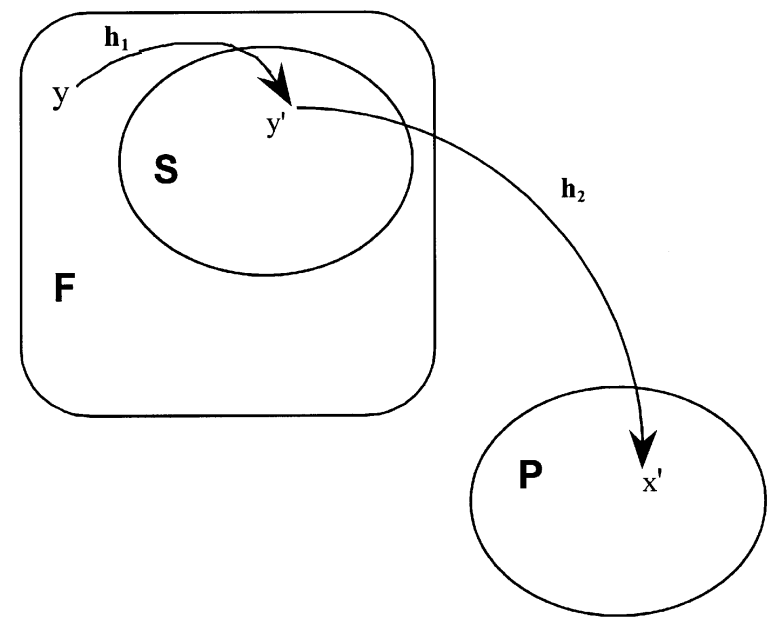

Fig. A1. Representation of the application $h_{1}$ and $h_{2}$.

Returning to the example developed in this work, in which space 7 is of dimension four, and $\boldsymbol{P}$ of dimension three, in the space $\boldsymbol{Z}$ exists a three dimensional subspace $\boldsymbol{S}$ from which we can extract a base expressed in reference to $\left\{f_{1}, f_{2}, f_{3}, f_{4}\right\}$ (the canonical base of 7 ).

$$
\exists \stackrel{h_{1}}{\longrightarrow} S \text { The application } h_{1} \text { being defined by the matrix: }[A]=\left\{\alpha_{i j}\right\} \text {. }
$$

There is consequently a surjective application $h_{1}$ of $\boldsymbol{Z}$ in $S$ which makes it possible to represent any element of $\boldsymbol{Z}$ in subspace $\boldsymbol{S}$, leading to determine a matrix of passage [A] of the application $h_{1}$ in space $\boldsymbol{Z}$ in subspace $\boldsymbol{S}$, definite by its coefficients.

$$
[\mathbf{A}]=\left[\begin{array}{llll}
\alpha_{11} & \alpha_{12} & \alpha_{13} & \alpha_{14} \\
\alpha_{21} & \alpha_{22} & \alpha_{23} & \alpha_{24} \\
\alpha_{31} & \alpha_{32} & \alpha_{33} & \alpha_{34}
\end{array}\right]
$$

\footnotetext{
${ }^{31}$ Thom R. (1972) Stabilité structurelle et morphogenèse. Édition Interédition.
} 
consequently:

$$
\left[\begin{array}{l}
y_{1}^{\prime} \\
y_{2}^{\prime} \\
y_{3}^{\prime}
\end{array}\right]=\left[\begin{array}{llll}
\alpha_{11} & \alpha_{12} & \alpha_{13} & \alpha_{14} \\
\alpha_{21} & \alpha_{22} & \alpha_{23} & \alpha_{24} \\
\alpha_{31} & \alpha_{32} & \alpha_{33} & \alpha_{34}
\end{array}\right]\left[\begin{array}{l}
y_{1} \\
y_{2} \\
y_{3} \\
y_{4}
\end{array}\right]
$$

The first stage in this work amount to expressing a base of the subspace $\mathbf{S}$ compared with the canonical base of 7 . The existence of this subspace is a priori the consequence of an established relation between the factors of contingency. Therefore, the hostility of the environment becomes at the first time the function of its degree of sophistication, of its complexity and size of the organisation ${ }^{32}$, and, to simplify it, this function can be regarded as linear, which makes it possible for example to consider:

$$
y_{2}=\left(a_{1}-a y_{1}\right)+b y_{3}+\left(c_{1}+c y_{4}\right) \text {. }
$$

Thus appears a subspace, of dimension inferior to space $\mathbf{7}$, in which are expressed all the observable combinations of the factors of contingency:

$$
\text { (I) } y=y_{1} e_{1}+y_{2} e_{2}+y_{3} e_{3}+y_{4} e_{4} \text {. }
$$

Where $\left\{e_{1}, e_{2}, e_{3}, e_{4}\right\}$ constitute the respective components of the canonical base of 7 from where:

$$
\text { (II) } y=y_{1}\left(e_{1}-a e_{2}\right)+y_{3}\left(b e_{2}+e_{3}\right)+y_{4}\left(e_{4}+c e_{2}\right)+\left(a_{1}+c_{1}\right) e_{2} \text {. }
$$

Compared to the new origin: $\mathrm{O}^{\prime}$ which leads in writing the new co-ordinate compared to the old base:

$$
\mathrm{O}^{\prime}=\left[\begin{array}{l}
0 \\
a_{1}+c_{1} \\
0 \\
0
\end{array}\right] \text { or: } \mathrm{O}^{\prime}=\left[\begin{array}{l}
0 \\
\gamma_{1} \\
0 \\
0
\end{array}\right] \text { with: } \gamma_{1}=a_{1}+c_{1} .
$$

And compared to this new reference mark:

$$
y=y_{1} s_{1}+y_{3} s_{2}+y_{4} s_{3}
$$

with: $\left\{\begin{array}{l}s_{1}=e_{1}-a e_{2} \\ s_{2}=b e_{2}+e_{3} . \\ s_{3}=e_{4}+c e_{2}\end{array}\right.$

\footnotetext{
${ }^{32}$ We implicitly consider that if the sophistication of the technical system increases that means (at least, initially) less hostility (with more cooperation agreements). If complexity increases, hostility will increase too (with members of the organisation having a more aggressive conception of the environment). Lastly, this same hostility will decrease following the size of the organisation.
} 
This new base, expressed to the canonical base of $\boldsymbol{Z}$, allow to specify the matrix $[\mathbf{A}]$ of the application $h_{1}$, indeed if we consider $y \in \mathcal{Z}$ and $y^{\prime} \in S$ we have:

$$
\left[\begin{array}{l}
y_{1} \\
y_{2} \\
y_{3} \\
y_{4}
\end{array}\right]=\left[\begin{array}{ccc}
1 & 0 & 0 \\
-a & b & c \\
0 & 1 & 0 \\
0 & 0 & 1
\end{array}\right]\left[\begin{array}{l}
y_{1}^{\prime} \\
y_{2}^{\prime} \\
y_{3}^{\prime}
\end{array}\right]+\left[\begin{array}{c}
0 \\
\gamma_{1} \\
0 \\
0
\end{array}\right]
$$

we express the matrix of the application $h_{1}$ :

$$
\left[\begin{array}{c}
y_{1}^{\prime} \\
y_{2}^{\prime} \\
y_{3}^{\prime}
\end{array}\right]=\left[\begin{array}{llll}
\alpha_{11} & \alpha_{12} & \alpha_{13} & \alpha_{14} \\
\alpha_{21} & \alpha_{22} & \alpha_{23} & \alpha_{24} \\
\alpha_{31} & \alpha_{32} & \alpha_{33} & \alpha_{34}
\end{array}\right]\left[\begin{array}{c}
y_{1} \\
y_{2}-\gamma_{1} \\
y_{3} \\
y_{4}
\end{array}\right]
$$

and, consequently:

$$
[\mathbf{A}]=\left[\begin{array}{lll}
1+a \alpha_{12} & \alpha_{12}-b \alpha_{12} & -c \alpha_{12} \\
a \alpha_{21} & \alpha_{22} & 1-b \alpha_{23}-c \alpha_{22} \\
a \alpha_{32} & \alpha_{32}-b \alpha_{32} & 1-c \alpha_{32}
\end{array}\right]
$$

\section{Appendix II: Construction of the matrix of passage $\boldsymbol{h}_{2}$}

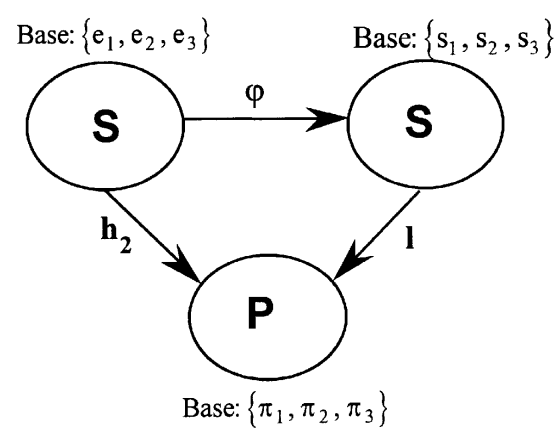

Fig. A2. Situation of the application $h_{2}$ in the model.

We seek to define the matrix of an application $g$ that enables expressing the components of a vector $x^{\prime} \in \mathcal{P}$ according to those of $y^{\prime} \in \mathcal{S}$ relatively to the base $\left\{\sigma_{1}, \sigma_{2}, \sigma_{3}\right\}$ :

$$
y^{\prime}=y_{1}^{\prime \prime} \sigma_{1}+y_{2}^{\prime \prime} \sigma_{2}+y_{3}^{\prime \prime} \sigma_{3}=x_{1}^{\prime \prime} \pi_{1}+x_{2}^{\prime \prime} \pi_{2}+x_{3}^{\prime \prime} \pi_{3} .
$$


From the application 1, we write the relation:

$$
l\left(y^{\prime}\right)=y_{1}^{\prime \prime} l\left(\sigma_{1}\right)+y_{2}^{\prime \prime} l\left(\sigma_{2}\right)+y_{3}^{\prime \prime} l\left(\sigma_{3}\right)=y_{1}^{\prime \prime} \pi_{1}+y_{2}^{\prime \prime} \pi_{2}+y_{3}^{\prime \prime} \pi_{3}=y^{\prime \prime} .
$$

The image by this application of a vector $x^{\prime}=x_{1}^{\prime \prime} \sigma_{1}+x_{2}^{\prime \prime} \sigma_{2}+x_{3}^{\prime \prime} \sigma_{3}$ becomes:

$$
l\left(x^{\prime}\right)=x_{1}^{\prime \prime} \pi_{1}+x_{2}^{\prime \prime} \pi_{2}+x_{3}^{\prime \prime} \pi_{3}=y^{\prime}=y_{1}^{\prime \prime} \sigma_{1}+y_{2}^{\prime \prime} \sigma_{2}+y_{3}^{\prime \prime} \sigma_{3} .
$$

We thus notice that relatively to the base $\left\{\sigma_{1}, \sigma_{2}, \sigma_{3}\right\}$, the components of $y^{\prime}=\left(y_{1}^{\prime \prime}, y_{2}^{\prime \prime}, y_{3}^{\prime \prime}\right)$ are expressed in relation to $\left(x_{1}^{\prime \prime}, x_{2}^{\prime \prime}, x_{3}^{\prime \prime}\right)$; with:

$$
\left[\begin{array}{l}
y_{1}^{\prime \prime} \\
y_{2}^{\prime \prime} \\
y_{3}^{\prime \prime}
\end{array}\right]=\left[\begin{array}{lll}
\lambda_{11} & \lambda_{12} & \lambda_{13} \\
\lambda_{21} & \lambda_{22} & \lambda_{23} \\
\lambda_{31} & \lambda_{32} & \lambda_{33}
\end{array}\right]\left[\begin{array}{l}
x_{1}^{\prime \prime} \\
x_{2}^{\prime \prime} \\
x_{3}^{\prime \prime}
\end{array}\right]
$$

which lead us to the result

$$
\left[\begin{array}{l}
x_{1}^{\prime \prime} \\
x_{2}^{\prime \prime} \\
x_{3}^{\prime \prime}
\end{array}\right]=\left[\begin{array}{lll}
\lambda_{11} & \lambda_{12} & \lambda_{13} \\
\lambda_{21} & \lambda_{22} & \lambda_{23} \\
\lambda_{31} & \lambda_{32} & \lambda_{33}
\end{array}\right]^{-1}\left[\begin{array}{l}
y_{1}^{\prime \prime} \\
y_{2}^{\prime \prime} \\
y_{3}^{\prime \prime}
\end{array}\right]
$$

where:

$$
\left[\mathbf{y}^{\prime}\right]=[\mathbf{L}]\left[\mathbf{x}^{\prime}\right] \Leftrightarrow\left[\mathbf{x}^{\prime}\right]=[\mathbf{L}]^{-1}\left[\mathbf{y}^{\prime}\right] .
$$

However with regard to the preceding results one can consider (by noticing that the matrix $[\Sigma]$ is regular):

$$
[\mathbf{L}]=[\Pi][\Sigma]^{-1} .
$$

Consequently:

$$
[\mathbf{L}]^{-1}=[\Sigma][\Pi]^{-1} .
$$

Thus at any point $y^{\prime} \in \mathcal{S}$ expressed compared to the basis $\left\{\sigma_{1}, \sigma_{2}, \sigma_{3}\right\}$ of this subspace, one associates it by the bijective application $g=l^{-1}$, a point $x^{\prime} \in \mathcal{P}$, expressed compared to this same base $\left\{\sigma_{1}, \sigma_{2}, \sigma_{3}\right\}$

$$
x^{\prime}=l^{-1}\left(y^{\prime}\right)=g\left(y^{\prime}\right)
$$

Let us define the application $h_{2}: S \stackrel{h_{2}}{\longrightarrow} \mathcal{P}$ starting from its matrix $[\mathbf{H}]$ and relatively to the base $\left\{e_{1}, e_{2}, e_{3}\right\}$. Let us consider first the automorphism $\varphi: S \stackrel{\varphi}{\longrightarrow} \mathbf{S}$ of the canonical base $\left\{e_{1}, e_{2}, e_{3}\right\}$ towards the base $\left\{\sigma_{1}, \sigma_{2}, \sigma_{3}\right\}$. The matrix of passage from one base to another will be the matrix $[\Sigma]$. This matrix will be that of automorphism $\varphi$, which results in:

$$
\varphi\left(e_{i}\right)=\sigma_{i}
$$

We thus write:

$$
y^{\prime}=y_{1}^{\prime} e_{1}+y_{2}^{\prime} e_{2}+y_{3}^{\prime} e_{3}=y_{1}^{\prime \prime} \sigma_{1}+y_{2}^{\prime \prime} \sigma_{2}+y_{3}^{\prime \prime} \sigma_{3} .
$$


That is to say $x^{\prime}$ the image in $\mathcal{P}$ of $y^{\prime} \in \mathcal{S}$ relatively to the base $\left\{\sigma_{1}, \sigma_{2}, \sigma_{3}\right\}$ by the application $g$.

$$
x^{\prime}=g\left(y^{\prime}\right) \Leftrightarrow x_{1}^{\prime \prime} \sigma_{1}+x_{2}^{\prime \prime} \sigma_{2}+x_{3}^{\prime \prime} \sigma_{3}=x_{1}^{\prime} e_{1}+x_{2}^{\prime} e_{2}+x_{3}^{\prime} e_{3} .
$$

Let us consider finally the vector:

$$
y^{\prime \prime}=y_{1}^{\prime \prime} e_{1}+y_{2}^{\prime \prime} e_{2}+y_{3}^{\prime \prime} e_{3} .
$$

We notice that the image $y^{\prime \prime}$ by the automorphism $\varphi$ we obtain:

$$
\varphi\left(y^{\prime \prime}\right)=y_{1}^{\prime \prime} \sigma_{1}+y_{2}^{\prime \prime} \sigma_{2}+y_{3}^{\prime \prime} \sigma_{3}=y^{\prime}
$$

consequently:

$$
g\left[\varphi\left(y^{\prime \prime}\right)\right]=g\left(y^{\prime}\right)=x^{\prime} .
$$

If we consider now the automorphism $\varphi^{-1}$, we obtain:

$$
\varphi^{-1}\left\{g\left[\varphi\left(y^{\prime \prime}\right)\right]\right\}=\varphi^{-1}\left(x^{\prime}\right)=x_{1}^{\prime \prime} e_{1}+x_{2}^{\prime \prime} e_{2}+x_{3}^{\prime \prime} e_{3} .
$$

Putting:

$$
x^{\prime \prime}=x_{1}^{\prime \prime} e_{1}+x_{2}^{\prime \prime} e_{2}+x_{3}^{\prime \prime} e_{3}
$$

The application $h_{2}$ become:

$$
h_{2}=\varphi^{-1}\left\{g\left[\varphi\left(y^{\prime \prime}\right)\right]\right\} .
$$

We thus find the application of $\boldsymbol{S}$ in $\mathcal{P}$ relatively to the base $\left\{e_{1}, e_{2}, e_{3}\right\}, h_{2}$ will be thus the isomorphism which, relatively to this basis, associates to the vector $y^{\prime \prime}$, the vector $x^{\prime \prime}$

$$
h_{2}\left(y^{\prime \prime}\right)=x^{\prime \prime}
$$

with

$$
h_{2}=\varphi^{-1} \circ g \circ \varphi \text {. }
$$

We interpret the application $h_{2}$ as the isomorphism of subspace $\boldsymbol{S}$ in space $\mathcal{P}$ relatively to the base $\left\{e_{1}, e_{2}, e_{3}\right\}$. The matrix $[\mathbf{H}]$ of this isomorphism will be written:

$$
[\mathbf{H}]=[\Sigma]^{-1}[\mathbf{L}]^{-1}[\Sigma]
$$

with

$$
[\mathbf{L}]^{-1}=[\Sigma][\Pi]^{-1}
$$

therefore

$$
[\mathbf{H}]=[\Sigma]^{-1}\left\{[\Sigma][\Pi]^{-1}\right\}[\Sigma]
$$

consequently the expression of the matrix:

$$
[\mathbf{H}]=[\Pi]^{-1}[\Sigma] .
$$




\section{References}

Chandler A. (1988) La main visible des managers. Economica, Paris.

Chandler A. (1989) Stratégies et structures de l'entreprise. Les Éditions d'Organisation, Paris.

Crozier M., Friedberg E. (1977) L'acteur et le système. Seuil, Paris.

Labourdette A. (1992) Théorie des organisations. Presses Universitaires de France, Paris.

Lawrence P., Lorsch J. (1989) Adapter les structures de l'entreprise. Les Éditions d'Organisation, Paris.

Miller D. (1993) Le paradoxe d'Icare. Comment les entreprises se tuent à réussir. Éditions ESKA, Paris.

Mintzberg H. (1982) Structure et dynamique des organisations. Les Éditions d'Organisation, Paris.

Morgan G. (1989) Images de l'organisation. Les Presses de l'Université de Laval, Éditions ESKA, Paris.

Simon H.A. (1991) Sciences des systèmes Sciences de l'artificiel. Éditions Dunod, Paris.

Thom R. (1972) Stabilité structurelle et morphogenèse. Édition Interédition, Paris.

Weber M. (1971) Économie et société. Plon, Paris.

Weber M. (1992) Essai sur la théorie de la science. Édition Plon, Collection Agora, Paris.

Woodward J. (1965) Industrial organization. Theory and practice. Oxford University Press, Oxford.

Zeeman C. (1977) Catastrophe Theory. Addison-Wesley, Boston.

To access this journal online: www.edpsciences.org 